\title{
ESTIMATION OF THE POLISHING TIME FOR DIFFERENT METALLIC ALLOYS IN SURFACE TEXTURE REMOVAL
}

Hernán A. González Rojas a , Antonio J. Sánchez Egea a , J. Antonio Travieso-Rodríguez ${ }^{\text {, }}$ Jordi Llumà i Fuentes ${ }^{c}$, Jordi Jorba Peiróc

${ }^{a}$ Department of Mechanical Engineering (EPSEVG), TECNOFAB group, Universitat Politècnica de Catalunya, Spain

${ }^{\mathrm{b}}$ Department of Mechanical Engineering (EEBE), DEFAM group, Universitat Politècnica de Catalunya, Spain

${ }^{c}$ Department of Materials Science and Metallurgical Engineering (EEBE), DEFAM group, Universitat Politècnica de Catalunya, Spain 


\begin{abstract}
The aim of this work is to propose a novel analytical model for predicting the polishing time and behavior of the surface texture removal in different metal alloys. The surface texture, resulting from a previous milling process, is characterized and investigated by the Abbott-Firestone curve, the relative speed of the abrasive material, the applied force, the type of material and the size of the abrasive grains. Consequently, a model that predicts the surface texture evolution based on the mechanism of abrasion is proposed, in which a constant of the wear model is found to behave linearly with the size of the abrasive grain for each metal alloy. Based on the good agreement between the experimental and the estimated values ( $\mathrm{R}^{2}$ equal to 0.993$)$, operational parameters are recommended to predict the required surface texture for $\mathrm{AlCu} 4 \mathrm{PbMg}, 30 \mathrm{CrNiMo8}$, C45E and X6CrNiMo8 when using abrasive grade between P180 and P1200.
\end{abstract}

Keywords: polishing model, material removal rate, Abbott-Firestone, surface texture

\title{
Introduction
}

The last manufacturing step for accurate products is the polishing process, which focuses to improve the material surface quality. The aerospace, automotive and biomedical sectors often demand to manufacture some parts or workpieces that require a mirror polished surface and the smaller geometric profile deviation [1] and [2]. In general, the polished workpieces exhibit an exceptional accuracy and good surface finish [3]. However, for complex geometries, the polishing process is usually applied manually by using several types of abrasives. Therefore, there is a lack of control over the removed material, and subsequently affects the desired dimensional accuracy [4]. Moreover, the finishing process is a very time consuming process, despite the fact that this process only modifies the surface profile in a micro scale level. To minimize the time spent in the process, several systematic methods have been developed to take into account the critical surface roughness variables and the volume of removed material, in order to identify which variable is more sensitive to reduce the polishing time, as stated by Lee et al. [5].

In general, polishing process employs glued abrasive particles, while buffing process uses free abrasive particles suspended in a liquid or wax medium. The finishing is 
considered the last step of the polishing process and is defined by an abrasive mechanism. In this case, a working pressure is applied using a pad or soft cloth with the purpose to change the irregular surface texture into a smooth surface texture. Marinescu et al. [6] described that, in order to obtain a good surface finishing, a low material removal rate $Q$ and a long polishing time are needed. Evans et al. [7] described the four most commonly accepted hypotheses in relation to the physical mechanism of material removal in the finishing process, which are: the abrasion hypothesis, the flow hypothesis, the chemical hypothesis and the friction wear hypothesis.

A lot of studies have been conducted in finishing the glass surface with a chemical mechanical process (CMP). The study of factors that characterize the CMP has generated various hypotheses and models. The first known study of polishing glass was made by Preston [8], developing the classical theory of wear. Other studies developed di erent models to estimate the material removal rate, for example Wang et al. [9] proposed a model that describes the relation between the parameters and the material removal rate of polishing. This model was relied on the probability of contact between the pad and the textured surface to be polished. The probability depends on the thickness of the fluid film located between both surfaces. Brinksmeier et al. [10] proposed a grinding process for finishing optical elements using a tool made of polyamide. The material removal rates were determined using the equation of Preston. They demonstrated that this new tool increases the material removal rate when the pressure and the polishing speed increase. Tsann [11] developed an analytic model to estimate the material removal rate during the polishing. This model was based on the elasto-plastic contact between the abrasive particles and pad. Therefore, this model goes one step forward to the model proposed by Preston. In addition, Savio et al. [12] developed a model for polishing glass based on the Reye's wear hypothesis, in order to predict the surface roughness depending on several operating parameters. The model shows the evolution of surface roughness during the polishing process, whereas the experimental results were validated by Preston [8]. However, Savio et al. [12] stated that the basic mechanisms of material removal in CMP are not well understood.

Recently, Jin et al. [13] developed a statistical model to predict the removed material by mechanical polishing. He described two types of abrasive particles: type I, particles that rotate and slide between the pad and the workpiece and type II, abrasive particles that 
are between the pad and the workpiece. If the abrasive grain size is small, the removed material is mainly due to type II. In contrast, when the grain size is large, the removed material is mainly due to type I. Tam et al. [14] developed another model that described how the strategy of material removal rate affects finishing surface geometry. To do that, different polishing pads were made by non-spherical abrasive tools, showing that the surface finish is highly dependent on the used pad. Following this last work, this paper develops a model for predicting the polishing time for textured surface metal alloys. This model estimates the polishing time when several variables are used. These studied variables are the abrasive-surface effective contact area, the surface profile, the type of material and the grain size of the abrasive paper, whereas the polishing speed and the polishing force remains constant. The proposed model is constructed on the analysis of the Abbott-Firestone curve to predict the final surface texture during the finishing process. The only material removal mechanism assumed in this model is the abrasion mechanism caused by the relative motion between the sand paper and the contactsurface of the material.

\section{Model}

The classical wear theory and experiments developed by Preston [8] describes that the material removal volume per unit of time $(Q)$ is proportional to the energy dissipated per unit of time. Moreover, the dissipated energy is a function of the cutting speed $\left(V_{c}\right)$ and the frictional force between the abrasive and the material. Then, the cutting speed is the maximum relative velocity between the abrasive and the material. Assuming that the frictional force is proportional to the polishing force $\left(F_{p}\right)$, which is acting normal to the surface, the material removal rate can be reduced to:

$$
Q=K \cdot V c \cdot F_{p}
$$

where $K$ is the proportionality constant.

Figure 1 shows the theoretical polishing of a surface previously machined following a parallel milling strategy with a ball end cutter, feed rate $(f)$ and sidesteps $(b)$. This previous machine process generates a surface pattern characterized by semicircular canals of period $b$. 


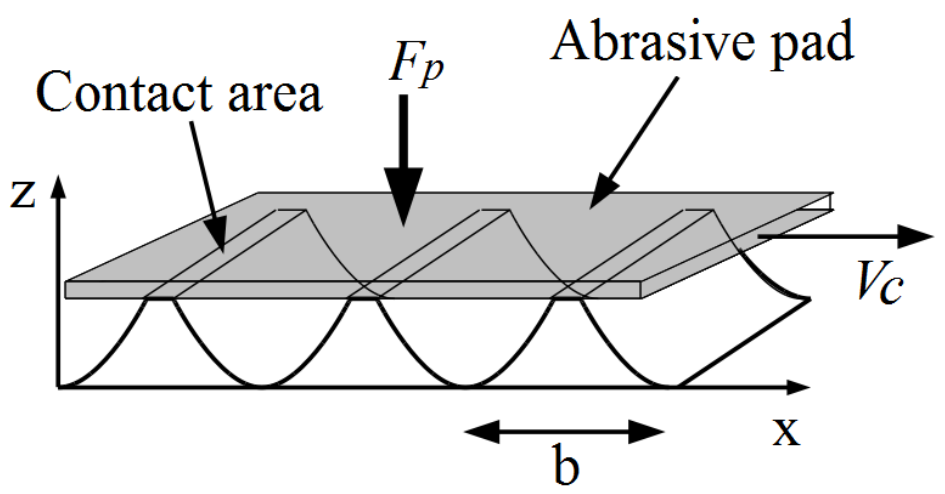

Figure 1: Theoretical polishing evolution of a milled surface.

Additionally, the material removal rate is the product of the polishing feed, multiplied by the effective contact area $(A c)$, or what is the same:

$$
Q=-\frac{d z}{d t} \cdot A c
$$

Replacing equation (2) into (1), an equation that predicts the planar surface position of polished feed $(z)$ is obtained.

$$
\frac{d z}{d t}=-K \cdot V c \cdot \frac{F_{p}}{A c}
$$

The effective dimensionless contact area $(A(z))$ is a function of the bearing ratio of the material area and is defined by the Abbott-Firestone Curve, as it was stated by Stout [15]. The contact area can be interpreted as an accumulative probability function of the ordinate $z(x, y)$ for a certain evaluation area.

$$
A(z)=\frac{A c(z)}{A t}
$$

where $A t$ is the total contact area or effective contact area in $\mathrm{z}=0$.

Replacing equation (4) into equation (3), the new expression predicts the planar surface position as a function of the Abbott-Firestone curve among other variables.

$$
\frac{d z}{d t}=-\frac{K \cdot V c \cdot F_{p}}{A t} \cdot \frac{1}{A(z)}
$$

With the equation (5) and the initial condition define by equation (6) allow to determine how the planar surface position progresses in time for any bearing ratio function.

$$
z(t=0)=R t
$$


where $R t$ is the maximum peak-valley roughness.

In this model, the polishing problem relies explicitly on the operation conditions: cutting speed $V_{c}$ and polishing force $F_{p}$, but also in the type of abrasive and the mechanical properties of the material. All these parameters are summarized in the $K$ constant. Therefore, it is needed to determine experimentally the relationship of the $K$ for each specific material and type of paper sand used. To do that, first the surface texture created by prior milling is characterized by the Abbot-Firestone curve $A(z)$.

\section{Model constant determination}

To determine the model constant, a least squares fit of a particular analytic solution of equation (5) is performed. Figure 2 shows the surface texture, parallel channels orientation, used to determine the mentioned constant. The semicircular channel were conceived with a ball end cutter with a small feed rate compare with respect to the sidestep.

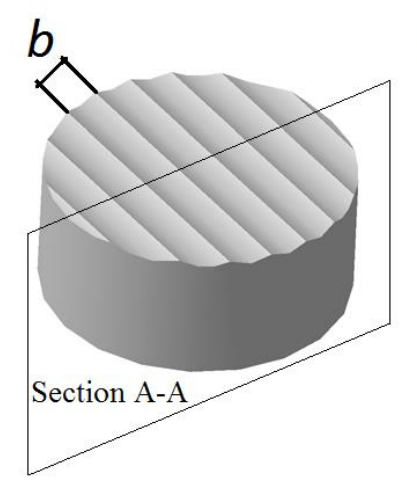

Section A-A:
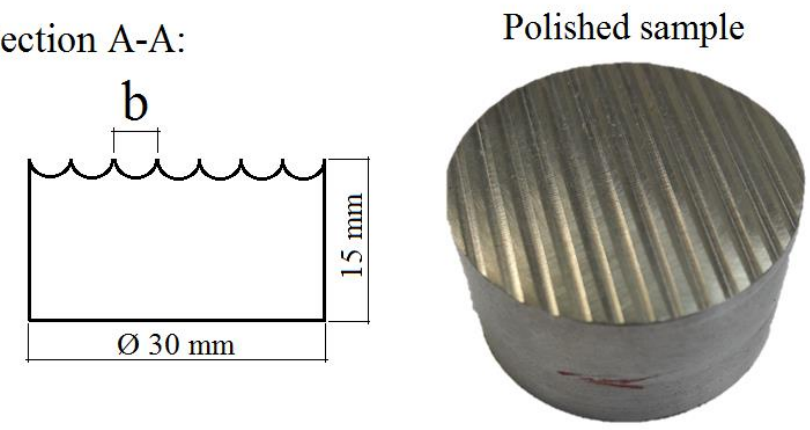

Figure 2: Machining of semicircular parallel pattern channels.

An analytical expression is deducted for the bearing ratio function of material area $A(z)$, when it is assumed a half period in the direction of the sidestep. In this case, the function which defines the surface texture is a small circular arc, and subsequently can be approximated by a parabolic curve with error $\mathrm{O}\left(x / r_{H}\right)^{2}$.

$$
z=\frac{x^{2}}{2 \cdot r_{H}}
$$

where $r_{H}$ is the radius of the ball end cutter. 
As the effective contact area depends on ordinate $z$, hence the bearing ratio function of material area $A(z)$ or Abbott-Firestone curve depends also on ordinate $z$. This relationship is observed in Figure 3.

$$
A(z)=1-\frac{2}{b} \sqrt{2 \cdot r_{H} \cdot z}
$$

Figure 3 shows the maximum peak-valley roughness in the surface texture. This roughness value can be written as follows:

$$
R t=\frac{b^{2}}{8 \cdot r_{H}}
$$

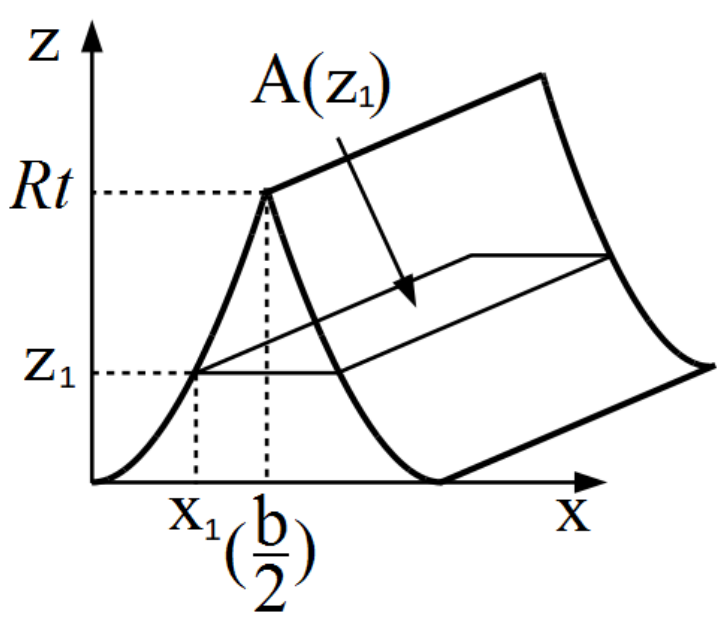

Figure 3: Approximation to the surface profile.

Substituting equation (9) into (8), an expression for the Abbott-Firestone curve of semicircular channel milling is found, such as those showed in Figure 2.

$$
A(z)=1-\sqrt{\frac{Z}{R t}}
$$

Replacing equation (10) in (5), the differential equation that predicts the advancing of the planar surface can be obtained. This dimensionless equation gives a compact expression that determines the plane surface texture, as shown in the following equation

$$
\frac{d z^{*}}{d t}=-\frac{P}{1-\sqrt{z^{*}}}
$$

where the dimensionless ordinate value $\mathrm{z}$ is

$$
z^{*}=\frac{Z}{R t}
$$

and the constant $P$ is 


$$
P=\frac{K \cdot V c \cdot F_{p}}{R t \cdot A t}
$$

The unit of constant $P$ is $\mathrm{s}^{-1}$. That is why the dimensionless time is defined by

$$
t^{*}=P \cdot t
$$

Finally, the polishing model (11) is completely defined by specifying the initial condition of the problem:

$$
z^{*}(t=0)=1
$$

In equation (13), the number $P$ allows to find the dimensionless time and thereby obtain a non-dimensional polishing model. This number is a fundamental part of this model, because number $P$ explicitly encloses working conditions such as cutting speed, polishing force and surface geometrical properties (the maximum peak-valley roughness $R_{t}$ and polished surface area $A_{t}$ ) which characterize the surface profile shown in Figure 3. On the contrary, the type of material and the type of abrasive are embedded in the $K$ constant.

Solving the analytic equation (11) with the initial condition (15), an equation that predicts the time evolution of the plane surface texture is shown below:

$$
\left(1-z^{*}\right)-\frac{2}{3}\left(1-z^{*} \frac{3}{2}\right)-P \cdot t=0
$$

Once the surface advance is known, an experimental data set $\left(z^{*}, t_{i}\right)$ is adjusted by least squares. The output value of the constant $\mathrm{P}$ is the best numerical fit of the experimental data to equation (16).

$$
\mathrm{P}=\frac{\sum\left(\left[1-\mathrm{z}^{*}-\frac{2}{3}\left(1-\mathrm{z}^{* \frac{3}{2}}\right)\right] \mathrm{t}_{\mathrm{i}}\right)}{\sum \mathrm{t}_{\mathrm{i}}^{2}}
$$

with $\mathrm{i}=1,2, \ldots . \mathrm{n}$, where $\mathrm{n}$ is the number of experimental points used in the fit.

Substituting (14) in (16) and clearing the variable $t^{*}$ with $\mathrm{z}^{*}$ equal to zero, the dimensionless polishing time to remove the surface texture left by a prior milling of semicircular channels is estimated as: 


$$
t^{*}\left(z^{*}=0\right)=\frac{1}{3}
$$

\section{Experiments and results}

The specimen dimensions were $30 \mathrm{~mm}$ diameter and $15 \mathrm{~mm}$ height. The initial surface texture is conceived by semicircular channels orientated in parallel patterns, as shown in Figure 2. The surface patterns are performed with the same milling tool and machining conditions: an $8 \mathrm{~mm}$ diameter ball end cutter, a spindle speed of $2500 \mathrm{rpm}$ and a feed rate $500 \mathrm{~mm} / \mathrm{min}$. Each pattern is defined by 3 cutting steps with a depth of cut of 0.5 $\mathrm{mm}$ each and a side-step of $2.2 \mathrm{~mm}$.

These specimens were polished automatically with a multidirectional polishing STRUERS LaboPol-5. During the experimental analysis of the planar surface advance, it was required successive measurements of the surface topography at different polishing times: $0,5,10,15,20,25$ and 30 seconds. Figure 4a shows the surface texture profile of X5CrNi18-10 polished with abrasive P180, at three different time instants $\mathrm{t}=0$ s, $15 \mathrm{~s}$, and $30 \mathrm{~s}$. The surface profile shown in Figure 4a was obtained with a Mitutoyo SJ-210 surface roughness diamond stylus profilometer. The value of the constant $P$ is obtained from the experimental points and equation (16). Figure $4 \mathrm{~b}$ shows the correlation between the experimental points and analytical model for the mentioned conditions. The repetition tests were different depending on the metal alloy: 6 specimens of aluminum, 4 specimens of stainless steel and 8 specimens for the two carbon steels were investigated. In all the specimens were performed 9 surface roughness measurements at different polishing times, consequently, a total of 54 points for $\mathrm{AlCu} 4 \mathrm{PbMg}$ aluminum and 36 points for each metal alloy (30CrNiMo8, X5CrNi1810 and $\mathrm{C} 45 \mathrm{E}$ ) were obtained to adjust the regression model.

The $K$ constant of the wear model has been determined on the basis of experimental data by using the equation (13). To achieve this, it is necessary to distinguish the cutting speed $V_{c}$ from the average relative speed between the workpiece and the abrasive, when a constant force $F_{p}$ is applied and a planar surface position $R_{t} z(\mathrm{t}=0)$ is defined. The order magnitude of these three parameters was: $V_{c}=0.33 \mathrm{~m} / \mathrm{s}, F_{p}=15 \mathrm{~N}$ and $R_{t}=0.12$ $\mathrm{mm}$. The abrasives paper sand used were discs of BUEHLER CarbiMet2 with granulometry of P180, P240, P320, P400, P600 and P1200. They are associated with 
average grain sizes $82,58.5,46.2,35,25.8$ and $15.3 \mu \mathrm{m}$ respectively. The polishing medium used during the experiments was water. These experimental conditions were studied in $\mathrm{AlCu} 4 \mathrm{PbMg}$ aluminum, 30CrNiMo8 alloy steel, X5CrNi18-10 stainless steel and $\mathrm{C} 45 \mathrm{E}$ carbon steel.

(a)



(b)

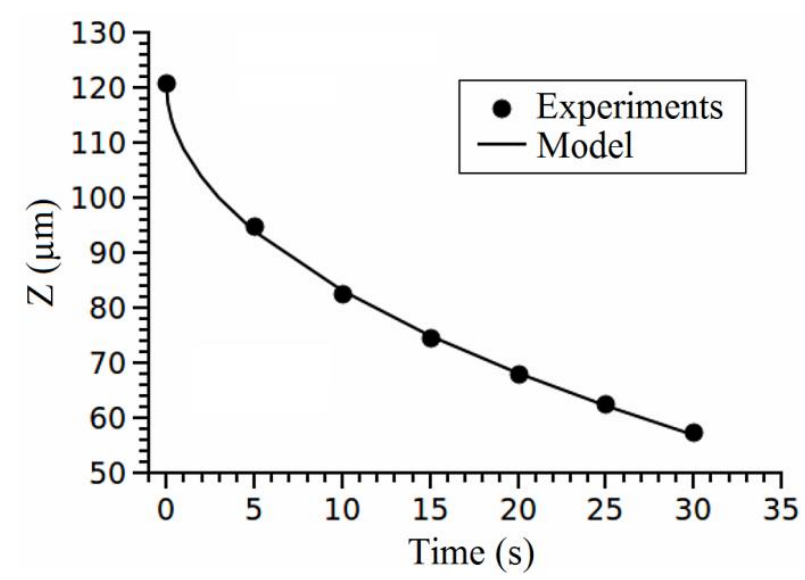

Figure 4: Polishing profile v/s time, (a) polishing profile measurement, (b) correlation between the experimental points and analytical model (line) for a 304 stainless steel workpiece polished with an abrasive pad of P240.

A set of experiments to study the effect of grain size and the type of metallic alloy on the surface finishing were performed. Figure 5 shows the relationship between the $K$ constant and the diameter of the particle or grain size $\left(D_{p}\right)$ in the four different materials tested. In general $K$ shows a linear behavior with respect to the variation of the grain 
size. If the grain size increases the $K$ constant increases and thereby increases the material removal rate. Moreover, each type of material presents an independent linear regression relationship between $K$ and $D_{p}$, as it is shown in Figure 5.

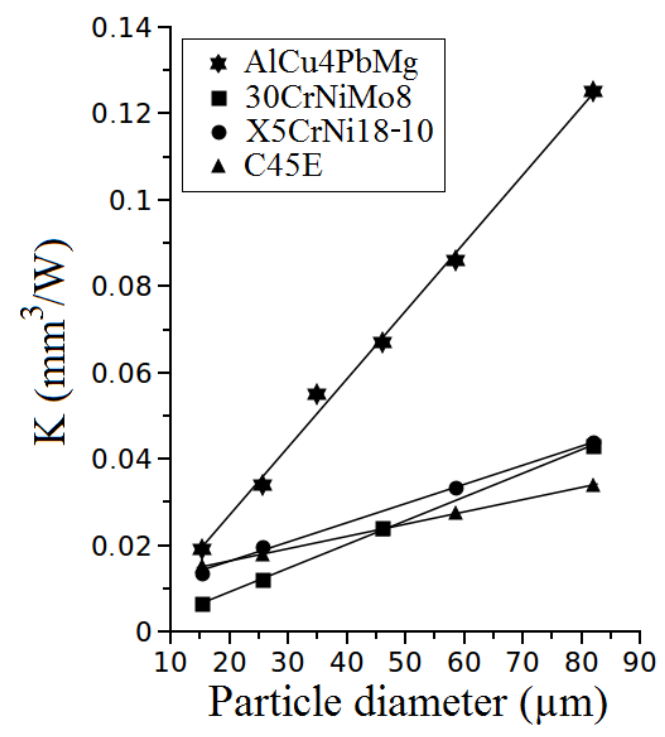

Figure 5: $K$ valor in function of $D_{p}$ to different metallic materials.

Table 1 shows the linear regression results for the four different metallic alloys. The coefficient $R^{2}$ adjusted is equal or greater than 0.993 for all the cases. Therefore, assuming that $\mathrm{K}$ has a linear behavior with respect to the variation of the abrasive grain size used is very reasonable.

Table 1: Material hardness and linear regression, $K\left(\mathrm{~mm}^{3} / W\right)$.

\begin{tabular}{ccccc}
\hline Material & $\mathrm{HV}$ & $\begin{array}{c}\mathrm{m} * 1000 \\
\left(\mathrm{~mm}^{3} / \mathrm{W} \mu \mathrm{m}^{-1}\right)\end{array}$ & $\begin{array}{c}\mathrm{K}^{*} 1000 \\
\left(\mathrm{~mm}^{3} / \mathrm{W}\right)\end{array}$ & $\begin{array}{c}\text { Adjusted } \\
\mathrm{R}^{2}\end{array}$ \\
\hline \hline AlCu4PbMg & 126.7 & $1.58 \pm 0.05$ & $-4.7 \pm 2.4$ & 0.993 \\
30CrNiMo8 & 385.5 & $0.55 \pm 0.01$ & $-1.9 \pm 0.4$ & 0.999 \\
X5CrNi18-10 & 202.6 & $0.44 \pm 0.01$ & $7,4 \pm 0.7$ & 0.994 \\
C45E & 384.9 & $0.25 \pm 0.00$ & $10.6 \pm 0.2$ & 0.999 \\
\hline
\end{tabular}

\section{Topography defined by the previous milling}

The previous milling defines the surface topography and plays an essential role on the planar surface advance. The surface profile effect has been introduced in the polishing 
model in terms of bearing material rate function $A(z)$. The equation (5) shows that the relationship between the planar surface advance and $A(z)$ are inversely proportional. If it is calculated the dimensionless values of equations (12), (13) and (14), using equation (5), an expression that estimate the dimensionless planar surface position is obtained. This equation depends on the bearing material rate and the dimensionless time.

$$
\frac{d z^{*}}{d t^{*}}=-\frac{1}{A\left(z^{*}\right)}
$$

The influence of the polishing time and the bearing rate is studied with a surface topography simulation. The surface topography simulation is defined by a theoretical milling process, using a ball end cutter for a parallel and cross mill strategies. This topography simulation assumes that the feed rate is small, while the removed material is defined by the geometrical intersection of the ball end cutter on the material. The theoretical computed topography is used to estimate the Abbott Firestone curve. The curve will be introduced at equation (19) to estimate the planar surface advance rate. This numerical approximation let us determine the relative effect of the lateral step movement on the planar surface advance rate. Figure 6 represents the surface topography made with 1:1 relative sidestep milling process. The simulation studied for this particular case represents a limit situation, when the feed rate is infinitely small and the previous milling marks were eliminated.

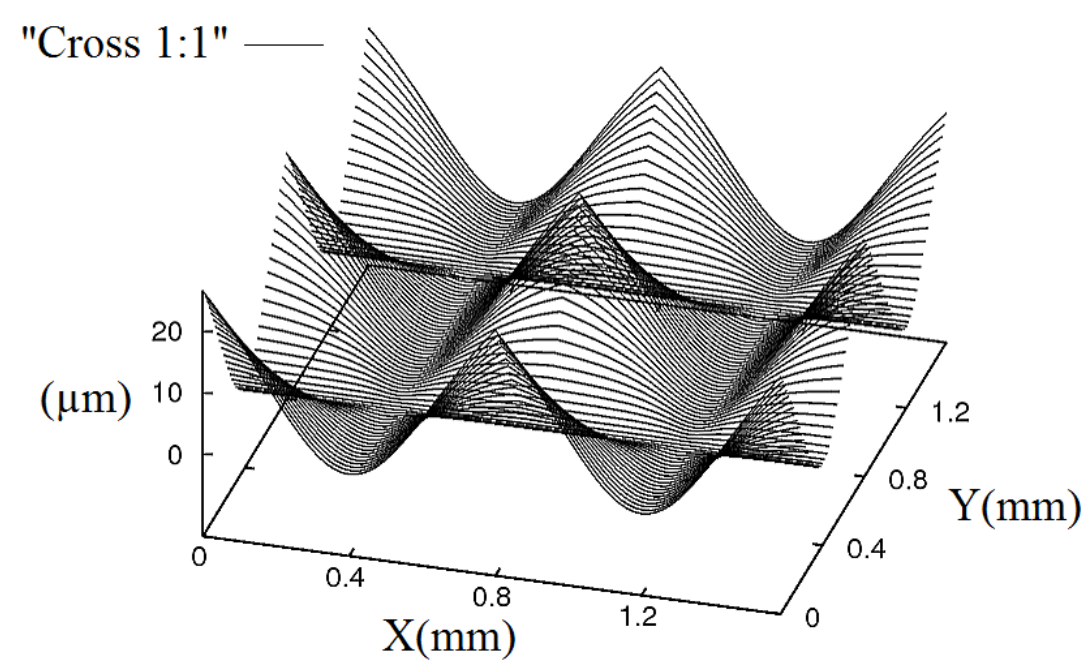

Figure 6: Surface topography of a cross milling done with a ball end cutter and relative lateral sidestep 1:1. 
Figure 7 shows the behavior of the Abbott-Firestone curve for different sidesteps of theoretical milling, defined in terms of the relation between $b x$ and $b y$, sidesteps on $X$ and on $Y$ respectively. These figures show the results of surface topographies made with a sidestep on bx:by of: 1:1, 5:1 and $\infty: 1$ (or parallel canals). Parallel canals can be understood as a limit relation between $b_{x}$ and $b_{y}$. If the relative lateral step increases, the bearing ratio increases. For example, a dimensionless position of the polishing profile $z^{*}$ equals to 0.4 , the bearing ratio for a relative steps $1: 1,5: 1$, and parallel are $0.20,0.29$ and 0.39 respectively.

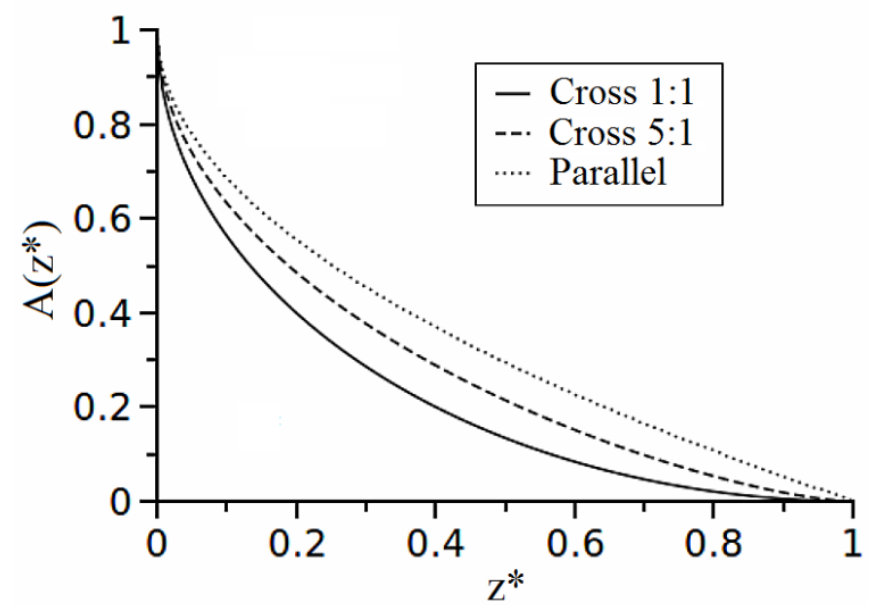

Figure 7: Abbott-Firestone curves for different cross-milling strategies.

Figure 8 shows the planar surface advance for the different size steps relations (for the three aforementioned machining strategies). In this figure, it is observed how the prior machining strategy influences on the polishing dimensionless time $t^{*}$. The polishing time required to remove the topography produced by prior milling $t^{*}$ max relies on the machining strategy selected. The cross milling strategies with sidesteps 1:1, 5:1 and parallel reach a polishing time $t_{\text {max }}^{*}$ of $0.21,0.27$ and 0.33 respectively. Therefore, the $1: 1$ cross milling strategy is the most favorable to minimize the polishing time. On the contrary, the parallel milling strategy is the worst condition to minimize the polishing time. 


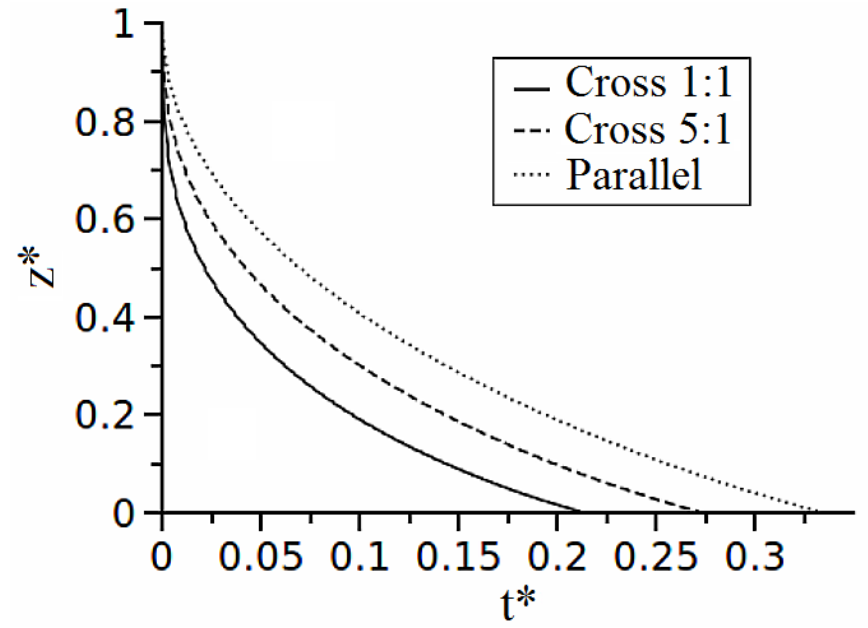

Figure 8: Planar surface advance for different prior milling.

To sum up, the model developed here allows the estimation of the polishing time required to remove topography left by any previous machining. In this context, it is necessary to calculate the bearing ratio of the topography defined by the AbbottFirestone curve. This novel model has proven an accurate prediction of the surface texture evolution for different metal alloys. The proposed model can be used either for a polishing process or a plateau-honing process. The implementation procedure of this process is to estimate the $K$ constant by polishing the surface texture of the workpiece and later using the regression equation (17). As a result, the bearing material rate function is obtained. To achieve that, the bearing material rate is replaced in equation (19), and, subsequently, by solving the output equation, an approximation of the surface texture behavior in function of the time step is obtained.

\section{Conclusions}

The conclusions of the paper can be stated as follows:

1. A model that predicts surface texture evolution based on the mechanism of abrasion has been successfully developed, in which the $\mathrm{K}$ constant of the wear model behaves linearly with the size of the abrasive grain for each metal alloy.

2. The model separately shows the contributions made by the abrasive grain size and the type of material from the contribution made by the prior milling. Therefore, the reference values of polishing time are estimated for two types of prior milling strategies: parallel and cross surface patterns for each material and abrasive grain size. 
3. From experiments, it was addressed that the prior machining strategy influenced on the polishing time. In particular, a 1:1 cross milling strategy is the most favorable to minimize polishing time, while the parallel milling strategy requires longer polishing time to remove the initial surface texture.

\section{Acknowledgements}

The authors gratefully acknowledge the Ministry of Economy and Competitiveness of Spain by financial support through the grant DPI2011-26326 (J-01686). The authors have no other professional and/or financial affiliations that may have biased the article.

\section{Nomenclature}

Ac Effective contact area

At Total contact area

$A(z) \quad$ Effective dimensionless contact area

$b \quad$ Sidesteps

$b x$, by Sidesteps in $\mathrm{x}$ and $\mathrm{y}$ axes respectively

$D_{p} \quad$ Grain size

$f \quad$ Feed rate

K Proportionality constant

$F_{p} \quad$ Normal polishing force

$P \quad$ Constant

$Q \quad$ Material removal volume per unit of time

$r_{H} \quad$ Radius of the ball end cutter

Rt Maximum peak-valley roughness

$t \quad$ Time

$t_{i} \quad$ Experimental polishing time points

$t^{*} \quad$ Dimensionless polishing time

$V_{c} \quad$ cutting speed

$W \quad$ Energy dissipated per unit of time

$z \quad$ Planar surface position

$z^{*} \quad$ Dimensionless ordinate value 


\section{References}

[1] Zhao, T.; Shi, Y.; Lin, X.; Duan, J.; Sun, P.; Zhang, J. (2014) Surface roughness prediction and parameters optimization in grinding and polishing process for IBR of aero-engine. Int J Adv Manuf Technol 74:653-663.

[2] Tsai, M.J.; Huang, J.F. (2006) Efficient automatic polishing process with a new compliant abrasive tool. Int J Adv Manuf Technol 30:817-827.

[3] Jain, N.K.; Jain, V.K.; Jha, S. (2007) Parametric optimization of advanced finishing processes. Int J Adv Manuf Technol 34(11-12):1191-1213.

[4] Dieste, J.A.; Fernandez, A.; Roba, D.; Gonzalvo, B.; Lucas, P. (2013) Automatic grinding and polishing using spherical robot. Procedia Eng 63:938-946.

[5] Lee, H.S.; Park, M.S.; Kim, M.T.; Chu, C.N. (2006) Systematic finishing of dies and moulds. International Journal of Machine Tools and Manufacture 46:1027-1034.

[6] Marinescu, I.D.; Rowe, W.B.; Dimitrov, B.; Inasaki, I. (2004) Tribology of abrasive machining processes. William Andrew Inc, Norwich, USA.

[7] Evans, C.J.; Paul, E.; Dornfeld, D.; Lucca, D.A.; Byrne, G.; Tricard, M.; Klocke, F.; Dambob, O.; Mullany, B.A. (2003) Material removal mechanisms in lapping and polishing. CIRP Annals-Manufacturing 52/2:611-633.

[8] Preston, F.W. (1927) The theory and design of plate glass polishing machine. Journal of the Society of Glass Technology 214-256.

[9] Wang, C-C.; Lin, S-C.; Hochen, H. (2002) A material removal model for polishing glass-ceramic and aluminum magnesium storage disk. International Journal of Machine Tools and Manufacture 42: 979-984.

[10] Brinksmeier, E.; Riemer, O.; Gessenharter, A. (2006) Finishing of structured surfaces by abrasive polishing. Precision Engineering 30:325-336.

[11] Tsann-Rong, L. (2007) An analytical model of the material removal rate between elastic and elastic-plastic deformation for a polishing process, Int $\mathbf{J}$ Adv Manuf Technol 32:675-681.

[12] Savio, G.; Meneghello, R.; Concheri, G. (2009) A surface roughness predictive model in deterministic polishing of ground glass moulds. International Journal of Machine Tools and Manufacture 49:1-7.

[13] Jin, X.L.; Zhang, L.C. (2012) A statistical model for material removal prediction in polishing. Wear 274/275:203-211. 
[14] Tam, H-Y.; Cheng, H. (2010) An investigation of the effects of the tool path on the removal of material in polishing. Journal of Materials Processing Technology 210:807-818.

[15] Stout, K.J. (2000) Development of methods for the Characterization of Roughness in three dimensions. Preston Press, London, UK. 